\title{
Note on Translation
}

Italics in Rayer's text indicate words and phrases emphasized by him. The footnotes to the translation are taken from the original 1840 French edition. Where Rayer used abbreviated book titles, these have been given in full. Other additions appear in square brackets. The full names and dates of writers from the sixteenth to the nineteenth century cited in these notes are given as far as possible in the Biographical Sketches. 